\title{
ANÁLISIS DE LA FORMACIÓN DE LAS ENFERMERAS IV. DE LA HIPÓTESIS DE TRABAJO Y LA RECONSTRUCCIÓN DE LOS ACTOS DE HABLA. LAS CONCLUSIONES FINALES.
}

Ana M. Piera Orts

\section{RESUMEN}

Se confirma la hipótesis de que el principio pedagógico de la conexión teoría - práctica se diluye ante las dificultades de integrar el modelo de formación con el modelo profesional asistencial y se va minimizando hasta la ruptura del mismo principio. Esta dificultad se halla en la base de la constitución de la relación institucional hospital-escuela, así como en la convivencia de dos modelos de ejercicio profesional: a) el ejercicio profesional como auxiliar del médico y b) el ejercicio como profesional que planifica cuidados de enfermería.

\section{EDUCATION OF REGISTERED NURSES:} A DIAGNOSIS

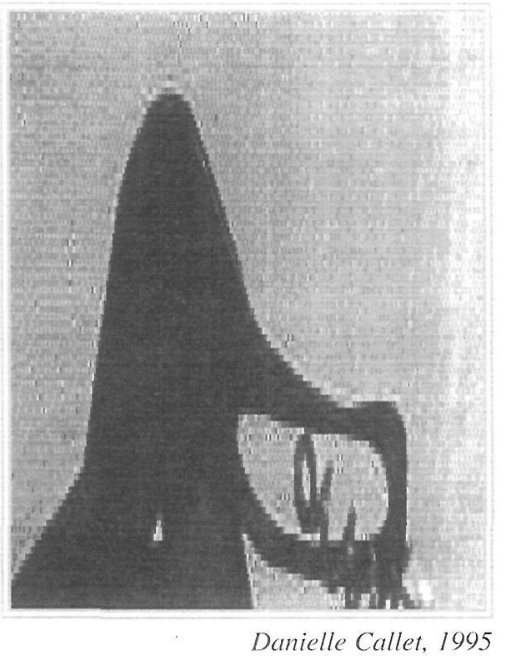

IV. A WORKING HYPOTHESIS. RECONSTRUCTION OF THE SPEECH. FINAL CONCLUSIONS.

\section{SUMMARY}

According to pedagogical education principles there should be a relationship between theory and practice. The present article proves that in the case of nursing education this is not so. The model used to educate nurses does not square up with the reality of being a professional nurse, there is a dichotomy between the student and the professional. Somewhere a rupture is produced. Perhaps the rupture occurs as a result of the simple difference between school - "theory"- and hospital - "practice", or may be due to the two different ways of understanding the nursing profession: i) the nurse as the physician's assistant, or ii) the nurse as the professional who plans nursing care.

\section{INTRODUCCIÓN}

En el recorrido realizado a través de los tres artículos anteriores, se ponía de manifiesto una situación de problematicidad en la formación básica de las enfermeras. Pusimos en marcha una serie de vías de acercamiento al tema y fuimos comprobando que los accesos se bloqueaban al no obtener las claves que producían el problema. Se inició pues una nueva vía: la obtención de datos de contraste a través de un cuestionario que permitió la confirmación de una hipótesis de trabajo: la ruptura del principio pedagógico teoría-práctica. Una hipótesis que sirvió de soporte para realizar un análisis empírico a través de la reconstrucción de los actos de habla, según la Teoría de la Acción Comunicativa, y desde donde se extraen conclusiones de relevancia en el orden institucional. 


\section{LA DE HIPÓTESIS DE TRABAJO.}

Cuando ya disponemos de la descripción histórica del momento en que surge la figura de la enfermera universitaria, del modelo de profesión bajo el que, generalmente, se actúa y del modelo de formación que parece ser adecuado, nos encontramos con un problema no definido, pero latente, a la vista de los comentarios recogidos en nuestras entrevistas, a lo largo de todo el proceso de nuestra indagación. Había problemas en la asignación de personal novel a los servicios profesionales; las enfermeras asistenciales diferían de las docentes en el valor que, en la actualidad, tienen las prácticas de las enfermeras-estudiantes y las enfermeras docentes consideran que en su trabajo tropiezan con gran número de dificultades. Había que modificar el esquema de trabajo descriptivo para buscar la vía que permitiese establecer una hipótesis de trabajo confirmada. Para la descripción de carácter reconstructivo de la totalidad cultural del grupo profesional de enfermeras docentes y asistenciales, el paso previo era examinar todas aquellas situaciones comunes o procesos de los grupos, así como los modos de percibir y explicar la realidad, para formular una descripción creible y posibilitarnos, posteriormente, discernir el detalle de la generalidad. De este modo, se amplía nuestro planteamiento que, inicialmente, era de corte cualitativo, para incorporar una herramienta cuantitativa, que nos posibilitase localizar algún tipo de relación categorial, elaborando un cuestionario de 48 items que se distribuyó por todo el territorio español y a partir de cuyos resultados se realizó un estudio correlacional, así como un análisis individual de cada item.

Para elaborar el cuestionario se tomaron, como referencia inicial, los materiales de entrevistas recogidos hasta el momento para examinar las posibles unidades de análisis. Posteriormente, se llevó a cabo una ronda de verificación de adecuación de los items. Así también, disponíamos de artefactos cualitativos (memorias docentes, programas docentes de prácticas, cuadernos de campo de los alumnos...) que nos permitirán incorporar datos de contraste.

Con técnicas no paramétricas, tratamos de recoger información procedente de un conjunto de población, que nos presentaba una gran dificultad para acceder a ella, pero, relevante para la confirmación de la línea de nuestra indagación. Si ciertamente entendemos que el enfrentamiento entre los métodos cualitativos y los cuantitativos está com- pletamente superado (COOK y REICHARDT, 1986; ALVIRA, 1983), la combinación metodológica, en nuestro caso, requiere que el tratamiento cuantitativo se realizáse igualmente, bajo una óptica descriptiva, de modo que localizásemos posibles relaciones sobre los conceptos que habíamos recogido e, incluso, el establecimiento de pruebas de hipótesis (MILLÁN, 1983; DANIEL, 1990).

Con los datos correlacionales, y sobre la base de lo revisado anteriormente, fue posible establecer una hipótesis de trabajo; siendo la hipótesis principal que el principio pedagógio de conexión teoría-práctica se diluye ante las dificultades de integrar el modelo de formación con el modelo asistencial y se va minimizando hasta la ruptura del mismo principio.

Esta hipótesis principal, se vincula a otras de rango menor, pero igualmente clarificadoras, como las que a continuación señalamos:

- Las creencias de los profesores de enfermería se manifiestan dispares cuando se hacen explícitos los modelos asistenciales, que influyen en la aplicación del principio pedagógico de conexión teoría-práctica.

- Las opiniones sobre la estructuración de las prácticas y su vinculación a la aplicación de cuidados y atenciones que se presentan como estudios de casos también se manifiestan dispares.

- Existe disparidad entre el profesorado en cuanto a la dirección de los conocimientos y la posible superposición de éstos debido a la modalidad organizativa del corpus teórico.

- Existe disparidad en la concepción de la organización didáctica de las prácticas, en orden a: se ajustan o no, los protocolos de actuación que se muestran en la escuela a las ejecuciones que se llevan a cabo en el centro sanitario donde se realizan las prácticas.

Con la reconstrucción de los actos de habla, centrados ya en una perspectiva cualitativa, tratamos de buscar qué tipo de construcciones intelectivas soportan la actividad docente, teniendo en cuenta los datos que hemos obtenido, a partir de los cuestionarios, como referencia.

La modalidad elegida para el análisis es la revisión del modo en que han sido configurados y organizados los conocimientos y las prácticas clínicas; lo que nos llevaría a reflexionar sobre diferentes aspectos, todos ellos en torno a ese mismo núcleo, pero que, por separado, nos alumbrarían sobre cuáles son los nexos que los articulan entre si. 
Esos aspectos pueden ser desde el modelo profesional que se deriva de las funciones establecidas en diferentes organizaciones o estatutariamente, hasta el modelo de enseñanza-aprendizaje, los vínculos organizativos entre hospital y escuela o la propia socialización de la enfermedad. De estos mismos aspectos, se derivarán necesariamente otros pero que, igualmente, los dirigiremos hacia el núcleo que nos interesa.

\section{LA CONFIGURACIÓN DE UNA RED DE IDENTIFICACIÓN CONCEPTUAL.}

El análisis no se ha planteado siguiendo los procedimientos analíticos generales de inducciones de categorías, comparaciones constantes, análisis tipológicos, enumeraciones o a partir de la elaboración de protocolos de observación (GOETZ y LECOMPTE, 1988), dada la dificultad en acotar algún elemento más concreto de lo que detectamos en las entrevistas y datos del cuestionario trabajado. Por ello, nos acercamos a los textos utilizando una revisión que mantiene las categorizaciones de un modo muy amplio.

Hay que situar, pues, el papel del investigador por las implicaciones teóricas que ello comporta, así como el modo de agrupamiento y análisis de los textos desde una perspectiva cualitativa que mantiene la pretensión de elaborar una construcción categorial mínimamente empírica.

El científico social, en su calidad de observador, recoge datos dependientes del lenguaje, por lo que ha de tener un status similar al del lego, al tiempo que sólo es factible que se abran al sujeto los significados si es participante, al menos virtual, en las comunicaciones de los miembros donde éstas se producen. Si ello no se produce de ese modo, no ha lugar al acceso posible a los nexos internos de sentido (HABERMAS, 1988), lo que viene a exigir que, el investigador social sea miembro integrante del grupo donde se producen las acciones comunicativas. Esta cuestión, en nuestro caso, asegura una situación razonable sobre el rol del investigador hermenéutico, quedando resuelta ésta, dada la condición de su pertenencia al entorno.

Con respecto al tipo de análisis, en este apartado se presenta un aspecto de la indagación que, siendo de naturaleza cualitativa, recoge empíricamente las construcciones teóricas que servirán para expresar el problema a estudio, a través de la meto- dología reconstructiva de los actos de habla al modo habermasiano. La cuestión se aborda, teniendo en cuenta que,

"las interpretaciones de las interpretaciones de la realidad se mueven en un nivel distinto que el de las interpretaciones de la realidad; en términos semánticos, los enunciados sobre enunciados son de orden distinto que los enunciados sobre hechos. Por otro lado, los contenidos semánticos tradicionales, que son los objetos de la investigación hermeneútica, son, a la vez, símbolos y hechos. Por lo tanto la interpretación es, también a la par, análisis empírico y análisis conceptual. Se dirige a los elementos de un mundo constituido mediante el lenguaje ordinario y a las propias reglas "gramaticales" que constituyen ese mundo" (McCARTHY, 1987, p.98).

y es precisamente el lenguaje ordinario, el terreno de esa intersubjetividad que hace posible la interacción y el entendimiento entre los individuos.

El análisis de las expresiones de la comunicación -con el procedimiento mayéutico como señala el propio Habermas- interroga una y otra vez al sujeto a partir de relaciones de semejanza, contrastes, paráfrasis y ejemplos, de modo tal que la relación de las teorías reconstructivas con el conocimiento cotidiano, no difieren de las ciencias empírico-analíticas como señala McCarthy (1987). Veamos que, si, dentro de las ciencias empírico-analíticas, lo que se hace es refutar el conocimiento preteórico de que se dispone a partir de los ámbitos objetuales para ser sustituido por explicaciones teóricas correctas, aunque con carácter provisional hasta nueva demostración en el terreno de las ciencias reconstrucctiva las hipótesis hacen explícito el saber preteórico y su relación con sus ámbitos objetuales.

En esencia, la teoría de la competencia comunicativa se basa en la reconstrucción de la base normativa del habla como sistema de pretensiones de validez universales y necesarias, atendiendo al principio básico de que el lenguaje no puede ser comprendido con independencia del entendimiento, sin el que no podríamos acceder al lenguaje mismo. Los resultados del análisis que se siguen de esta noción de teoría comunicativa, en la que entran en consideración, tanto actos de habla como acciones no-verbales, tienen para Habermas la finalidad de suministrar la base para una investigación social. El presupuesto básico, que descansa en esta teoría 
comunicativa, es el consenso generado a partir del recíproco planteamiento de los hablantes, del mutuo reconocimiento de cuatro tipos de validez.

En este nuevo marco y dado que nos fue posible establecer una hipótesis de trabajo, nuestro diseño emergente nos permitía la opción de buscar razones de naturaleza teórica o práctica, que nos acercasen a la comprensión de las hipótesis de menor rango y cuya relación directa con el principio pedagógico de la conexión teoría-práctica ya comentamos. Ahora, cabía la posibilidad de buscar esas razones y argumentos a partir del análisis de los textos recogidos en entrevistas abiertas y charlas, para seguir en la línea de nuestra indagación.

El bloque de entrevistas realizadas a un grupo de profesores de la Escuela de Enfermería, y que más tarde se confrontarían estas opiniones con las de personal asistencial, se planteó insistiendo en que no se trataba realmente de una entrevista abierta o no estructurada, sino que, con el ruego de que atendieran a las afirmaciones y preguntas que esta autoría formulaba, iniciasen una rueda de comentarios entre ellos, sobre lo que era fruto de nuestras reflexiones en torno a los contrastes obtenidos a través del cuestionario que vimos en el apartado anterior o la de algunos autores que habían tratado el tema; y a partir de estos actos de habla, trataría de estudiar los modos de su estructura autorreferencial, con el fin de localizar en qué aspectos los "actores tratan de sintonizar cooperativamente sus planes de acción en el horizonte de un mundo de la vida compartido y sobre la base de interpretaciones comunes de la situación" (HABERMAS, 1990a, p., 74). Esta búsqueda, naturalmente, se orienta a través del análisis del habla consensual, que ha de servir de base para el análisis de los modos deritativos y defectivos del habla como formas de comunicación estratégica o deformada.

Para poder contrastar el contenido proposicional de los actos de habla que fuimos recogiendo, además de la participación directa en la recogida de estos testimonios, lo que nos permitía revisar parte de la validez de las emisiones, disponíamos de unos documentos elaborados por los alumnos, con carácter colectivo, que nos servirían de contraste, al igual que de algunas entrevistas con enfermeras y profesoras, de las que disponíamos de grabaciones breves, pero sustantivas, ya que se realizaron a la vista de los materiales de prácticas de los alumnos, en las que se hace referencia directa a conversaciones con el profesorado responsable de las mismas.

Se efectuó un largo proceso reconstructivo identificando diferente tipo de afirmaciones como actos de emisión representativos, constatativos, regulativos o perlocucionarios y los posibles efectos deritativos que, en su estructura, exigen razones bien del discurso teórico o práctico o, en ocasiones, tan solo como modos expresivos de compartir un valor educativo dado.

\section{CONCLUSIONES.}

Para finalizar presentamos unas reflexiones a modo de conclusión de este trabajo, pero con la indicación que sólo se anota aquí la parte más relevante, pero en modo alguno la totalidad que alumbra la camplejidad de los revisado. La escasa tradición investigadora en torno a los modelos formativos de las enfermeras españolas fue derivando nuestra indagación por diferentes caminos. Pensábamos, inicialmente, que había una situación de formación deficitaria que podría achacarse a aspectos de diseño didáctico. Buscamos en el modo de institucionalización de sus enseñanzas y tropezamos con la trasposición de las enseñanzas médicas. Comparamos el modelo profesional con el modelo de formación y detectamos una falta de correspondencia. Posteriormente, creímos ver que el problema podría tratarse desde la óptica del pensamiento del profesor y cómo se enfrenta éste a la tarea de introducir al alumno en un mundo lleno de ambigüedades y nos encontramos con una escasa definición de su propia construcción curricular, entremezclada con cuestiones de naturaleza institucional, tales como la institucionalización del quehacer enfermero y la propia institucionalización de sus programas formativos, o el propio diseño de la carrera profesional ligada a la formación de ciclo único.

Cabe pensar sin grandes riegos de errar que esta profesión, como muchas otras, se halla en una situación de cambio, cuando todavía no tiene unos referentes de su propia autorregulación y cuyos procesos formativos, lejos de disponer de una amplia formalización, se hayan en la actualidad dentro de un marco educativo en el que otras profesiones, que se hallan muy próximas, están configuradas de modos mucho más concretos, como los técnicos especialistas, que siguen una estructura teórica similar a los programas de carácter propedéutico universitario, en los que las prácticas se organizan en torno a conocimientos concretos especializados y acotados 
a áreas muy específicas como técnicas de laboratorio, de radiología o anatomía patológica, sin trato directo con el cliente-paciente, y que han venido ganando espacio al ejercicio enfermero.

Si sólo se tratase de exponer el resultado de una indagación abierta, apuntaríamos algo muy breve: el ejercicio profesional de la enfermera, por su bajo estatuto científico y su escaso control profesional, implica que la formación de nuevos profesionales, dentro de ese marco, participe de la misma dinámica; manteniendo un círculo en el que no penetran principios pedagógicos tales como la conexión teoría-práctica, que permitiría la adecuación de los nuevos conocimientos dentro de los formatos didácticos como elementos de trasmisión del conocimiento profesional.

Sin embargo, nos hemos encontrado con una situación nada fácil de definir, en la que operan muchos factores que afectan al ejercicio enfermero y, por tanto, a la formación de sus miembros. Aspectos que, ahora, dejamos perfilados apuntando hacia nuevas indagaciones y trabajos más acotados, que posibiliten un mejor conocimiento y una búsqueda de alternativas a cada una de las situaciones dilemáticas que se pudieran definir.

Nuestro trabajo, siempre de naturaleza exploratoria, queda configurado como un acercamiento a las dificultades que profesores y personal de enfermería encuentran en el proceso de formación y socialización de sus nuevos miembros, tratando de definir una situación problematizada; toda vez que se vierten afirmaciones que muestran el proceso de formación cargado de inconsistencias en cuanto a los modelos asistenciales se refiere, así como su conexión desde la vịda académica de la enfermera.

Para ello presentamos una serie de cuestiones, que, aunque unidas entre sí, nos permiten mostrar una serie de relaciones que definen el conjunto de los problemas que hemos tratado de configurar en este trabajo:

- Se dan dos modelos de ejercicio profesional, es decir, dos modos diferentes de ver el papel de la enfermera: el ejercicio de una labor informada e independiente y el ejercicio vinculado a la figura del facultativo y dependiente de éste.

- El ejercicio profesional enfermero no gobierna su propia regulación y tampoco decide sobre la totalidad de sus funciones
- La autoimagen que las enfermeras poseen de su trabajo, aunque es importante para ellas, a juicio de éstas, no trasciende a los sujetos ajenos a su profesión

- E1 bajo estatuto científico de sus aplicaciones técnicas, que muestran a través de sus afirmaciones, creemos que guarda una estrecha relación con la consideración de tratarse de una profesión eminentemente femenina.

- La organización de las clases teóricas y prácticas, no cuenta, realmente, con una estructura adecuada para el ejercicio docente, en la medida que los profesionales en ejercicio asistencial no están integrados en los programas de formación, exceptuando la figura del profesor asociado de prácticas.

- Una dificultad derivada de un concepto ausente: el hospital es la escuela y la escuela es el hospital, con la extensión necesaria al Centro de Salud o cualquier otra organización de servicio sanitario que sea fuente de experiencias reales.

- La conexión teoría-práctica se presenta de un modo superficial, denominando teoría tan sólo a aquellos aspectos que formalmente recogen los manuales y práctica, a aquello que comporte una actividad física, empobreciéndose así este binomio, cuyo sentido real sería ver-cómo y actuar-como, permitiendo al sujeto percibir las situaciones nuevas y problemáticas no familiares, como si lo fueran (SCHÖN, 1992) y posibilitando un aprendizaje de ida y vuelta, que circulase entre el discurso teórico y el práctico.

Esta situación no tiene una solución fácil, pero tampoco es imposible de abordar, al menos, en lo que se refiere a la coordinación completa entre administración y profesionales, como ya se demostró en 1933 cuando se contempló la creación de la Escuela Nacional de Enfermeras Visitadoras, aneja a la Escuela Nacional de Sanidad, con la colaboración de la Fundación Rockefeller en un programa de formación para aquellas futuras enfermeras-profesoras (BERNABEU MESTRE y GASCÓN PÉREZ, 1995); proyecto que se vería interrumpido tras la Guerra Civil Española y que jamás se volvió a retomar. Como ejemplo significativo, en los programas formativos de aquellas enfermeras se incluía una disciplina denominada "introducción a la sociología curricular", aspecto curioso, dado que este tipo de contenidos llega a ser, en algunos casos de profesores de prácticas, un concepto absoluta- 
mente ajeno a su ejercicio docente, como pudimos constatar tres largas entrevistas.

Otro aspecto de la planificación educativa, que se nos muestra muy directamente, es que ésta se reduce a un tipo de actividad docente de distribución de contenidos del área y de organización de tiempos como constatamos en el modelo de formación; pero en modo alguno pudimos percibir la existencia de un cierto nivel de formalización de las enseñanzas. En todo caso, sí percibimos la dificultad de "¿como enseñar la complejidad de situaciones que se dan en la práctica profesional?", como nos planteaba una de las profesoras que participó en nuestra charla compartida.

Desde luego, la muestra de ese reduccionismo en la planificación se detecta en el modo en que el modelo de formación aparece enfrentado al de socialización profesional. Es decir, lo que no se aprende de modo derivado de la presentación formal de los requisitos para el ejercicio profesional, bajo un modelo empírico, ecológico, integral o cualquiera que se estimase a priori, se adquiere dentro de ese mundo complejo en el que se aprende cómo fundir normas incompatibles o potencialmente incompatibles en un todo organizado de un modo consistente; secuencia que en principio sería posterior al proceso de formación, pero que en este ámbito, ambos modelos entran en manifiesta competición.

Las partidas presupuestarias que contemplan gastos de personal, formación continuada, ayudas para proyectos de investigación, tesis doctorales o nuevas tecnologías no tendrían que verse fuertemente incrementadas, al distribuir una pequeña parte de la plantilla, de carácter alternante, con horarios de trabajo que posibilitasen los círculos de reflexión, análisis de técnicas y conocimientos a partir de procesos participativos y compartidos con los profesores (KEMMIS y McTAGGART, 1988), de modo que todos los que lo deseasen pudiesen participar en estos programas de mejora, que comportarían un punto de apoyo para la estrecha colaboración con los responsables de la formación básica de enfermeras que fuera en ambos sentidos ente escuela y hospital.

Los contenidos formativos deberían quedar garantizados no sólo por su consistencia y efectividad, sino también, por su adecuación a la realidad profesional y por la coordinación institucional, que fuesen más allá de la simple garantía de acceso al área asistencial, para realizar prácticas y diesen ocasión a los alumnos al acercamiento a un mundo profesional en el que se producen situaciones singulares.

\section{BIBLIOGRAFÍA*}

ALVIRA MARTÍN, F. (1983) Perspectiva cualitativa perspectiva cuantitativa en la metodología sociológica. En: Revista Española de Investigaciones Sociológicas. núm. 2. págs. 5375.

APPLE, M.W. (1986) Ideología y Currículo. Trad. por R. Lassaletta. ed.original. 1979. Madrid. Ediciones Akal. págs. 224.

ARDOINO, J. (1980) Perspectiva política de la educación. Trad. por A. Casais. Narcea, S.A. de Ediciones. p. 311.

BERNABEU MESTRE, J.; GASCÓN PÉREZ, E. (1995) El papel de la enfermería en el desarrollo de la salud pública española (1923-1935) la visitadora sanitaria. En: Dynamis. Acta Hisp. Med. Sci. Hist. Illus., 15, 151-176. COOK T.D. Y REICHARDT, CH. S. (1986) Hacia una superación del enfrentamiento entre métodos cualitativos y los cuantitativos. En:COOK y REICHARDT. Métodos cualitativos y cuantivativos en investigación evaluativa. Madrid. Ed. Morata. trad. G.Solana.ed.orig. 1982. pp. 2558.

DANIEL, W.W. (1990) Bioestadística. Base para el análisis de las ciencias de la salud. Ed. Limusa. México. pp. 667.

FOUCAULT, M. ( 1991 ) El nacimiento de la clínica. Una arqueología de la mirada médica.Siglo Veintiuno de España Editores, S.A. $14^{a}$ edición. Ed.. original 1963. pp. 293.

GOETZ, J. P. y LeCOMTE, M. D. (1988) Etnografía y diseño cualitativo en investigación educativa. Ed. original 1984. Ediciones Morata, S.A. pp. 279.

HABERMAS, J. (1990a) La lógica de las ciencias sociales. Trad. por M.Jimenez. $2^{\mathrm{a}}$ ed. castellano. ed. original 1982. Madrid. Ed. Tecnos. p. 506.

KEMMIS, S., McTAGGART, B. (1988) Cómo planificar la investigaciónacción. Trad. por Salcedo, sobre la Tercera ed. revisada de 1987. Barcelona. Laertes S.A. de Ediciones. p. 199.

McCARTHY, T. (1987) La Teoría Crítica de Jürgen Habermas. Ed. Tecnos, S.A. pp. 479

MILLÁN VENTURA, M. (1983) Estadística aplicada a las ciencias humanas. Ed. Promolibro. Valencia. p, 355. PEYA GASCÓN, M. (1991) La formación en Enfermería. En: Todo Hospital, núm. 74. pp. 35-41.

SCHÖN, D.A. (1992) La formación de profesionales reflexivos. Hacia un nuevo diseño de la enseñanza y el aprendizaje en las profesiones. Ed. Paidós. MEC. pp. 310.

* Considérese la imposibilidad de presentar el volúmen de reseñas consultadas. Tan solo se citan las que aparecen en este breve texto. 